\title{
Valor pronóstico del aclaramiento de lactato en pacientes pediátricos críticos
}

\author{
Dante A. Fuentes-Mallozzi ${ }^{1 *}$, José D. Llanas-Rodríguez ${ }^{1}$ y Mauricio Peña-Equihua ${ }^{2}$ \\ ${ }^{1}$ Dirección General, Hospital Infantil de Tamaulipas; ${ }^{2}$ Hospital Regional de Alta Especialidad de Ciudad Victoria. Ciudad Victoria, Tamaulipas, México
}

\begin{abstract}
\end{abstract}
Background: Lactate clearance at $6 \mathrm{~h}$ appears to be a useful test that allows predicting mortality with adeguate sensitivity and specificity. We considered important to validate this test in Mexico. Objective: To determine the sensitivity $(\mathrm{Se})$, specificity (Sp), and area under the curve (AUC) of lactate clearance in a pediatric critically ill population $\overline{5}$ and compare it with the pediatric index of mortality (PIM 2). Methods: A prospective, multicenter, observationa analytical protocol was conducted. Patients $1 \mathrm{mo}-17 \mathrm{y} / 11 \mathrm{mo}$ old were enrolled at the Children's Hospital of Tamautipas (HIT) and the High Specialty Regional Hospital of Ciudad Victoria (HRAEV). Statistical analysis included designiing a $2 \times 2$ contingency table to determine the Se, Sp, negative (NPV) and positive (PPV) predictive values, ROC curvês for lactate clearance at $6 \mathrm{~h}$ with a cut-off of 10\%, and PIM 2. Both AUCs were compared using the method of Hannley and McNeil, establishing a value of $p \leq 0.05$ for statistical significance. Data were analyzed using SPSS ${ }^{\circledR}$ v $2 \stackrel{P}{ }$ and Epidat ${ }^{\circledR}$ v.3.0. Results: A total of 105 patients were included, $43(41 \%)$ male and $62(59 \%)$ female, with a mea of $65.2 \pm 67.1$ months; 12 (11.4\%) children died and 93 (88.6\%) survived. Results for lactate clearance were: Se $83.3 \%$, Sp 66.6\%, PPV 24.3\%, NPV 96.8\%, and AUC 0.75 ( $p=0.005$ ); PIM 2 AUC was 0.88 ( $p=0.000$ ). Hanley \& MẽNeilDeLong test was $p=0.1693$. Discussion: We obtained similar results for sensitivity and specificity as those foukd in the only meta-analysis reported to date. Furthermore, both AUC were alike. Therefore, we conclude that clearance of lactate at $6 \mathrm{~h}$ was similar to PIM 2 to predict mortality in critically ill pediatric patients. (Hosp Med Clin Manag. 2019/12:152-6) Corresponding author: Dante A. Fuentes-Mallozzi, danteph@hotmail.com

Key words: Prognostic. Lactate. Clearance.

\section{RESUMEN}

Introducción: El aclaramiento de lactato a las $6 \mathrm{~h}$ parece ser una prueba útil que permite pronosticar la mortaliđad con una adecuada sensibilidad y especificidad, por lo cual es importante validar estos resultados en México. Objetivo: Determinar la sensibilidad (S) y especificidad (E) del aclaramiento de lactato y área bajo la curva (ABC) en paciente§_pediátricos en estado crítico y compararlo con una escala pronóstica de mortalidad pediátrica. Métodos: Estudio prospéetivo, observacional, multicéntrico y analítico, realizado del 1 de febrero de 2015 al 31 de enero de 2016 en el Hospitaflnfantil de Tamaulipas (HIT) y el Hospital Regional de Alta Especialidad de Ciudad Victoria (HRAEV). Se incluyeron pacientes de 1 mes a 17 años y 11 meses de edad; se determinó el índice pediátrico de mortalidad (PIM 2) y el porcentaje

\section{Correspondence:}

*Dante A. Fuentes-Mallozzi

E-mail: danteph@ @otmail.com
Date of reception: 19-09-2018

Date of acceptance: 11-10-2019

DOI: 10.24875/HMCM.19000159 
de aclaramiento de lactato a las 6 h (CL6). Se realizó tabla de contingencia $2 \times 2$ para conocer la S, la E, el valorłpredictivo negativo (VPN) y el valor predictivo positivo (VPP). Se obtuvieron ambas ABC y se compararon con la pruebba de Hanley \& McNeil-DeLong, estableciendo un valor de $p \leq 0.05$. Se utilizaron los programas SPSS ${ }^{\circledR}$ v.21 y Epida $\AA_{-}^{\circledR}$ v.3.0. Resultados: De 105 pacientes, 43 (41\%) fueron del sexo masculino y 62 (59\%) del femenino. Media de edad: $65.2 \pm 67.1$ meses. Defunciones 12 (11.4\%), supervivientes 93 (88.5\%). Los resultados para el CL6 < 10\% fueron: S 83.3\%, E66.6\%, VPP 24.3\%, VPN 96.8\% y ABC de 0.75 ( $p=0.005$ ). Para el PIM 2: ABC 0.88 ( $p=0.000)$. Hanley \& McNeil-DeLong $\mathrm{p}=0.1693$. Discusión: Nuestros resultados son similares a lo reportado en la literatura y los valores de ambas: $\bar{A} B C$ no demostraron diferencia alguna entre ellas, por lo que el CL6 < 10\% es una prueba comparable al PIM 2.

Palabras clave: Pronóstico. Lactato. Aclaramiento.

\section{INTRODUCCIÓN}

El lactato ha demostrado ser útil como marcador pronóstico de mortalidad en el paciente tanto adulto como pediátrico en estado crítico; su aplicabilidad radica en que entre más alto sea el valor sérico obtenido, peor es el pronóstico del paciente ${ }^{1,2}$.

En el 2004 Nguyen, et al. ${ }^{3}$, pioneros en el estudio del aclaramiento o depuración del lactato, demostraron en el paciente adulto con sepsis y choque séptico que el aclaramiento de lactato a las $6 \mathrm{~h}$, con un punto de corte de $<10 \%$, es útil para predecir la mortalidad hospitalaria. Es a partir de este primer estudio que otros más se han realizado en pacientes adultos, los cuales han validado el aclaramiento de lactato como herramienta pronóstica de mortalidad en pacientes críticos de cualquier etiología, incluyendo aquellos con sepsis, trauma, neumonía, cardiopatías y muchas otras ${ }^{4-7}$.

En 2014 se realizó un metaanálisis de todos los trabajos publicados de pacientes adultos hasta ese momento, en donde se estudió el aclaramiento de lactato como prueba pronóstica de mortalidad, confirmando que entre menor sea el porcentaje de aclaramiento de lactato, mayores serán las posibilidades de morir o, a la inversa, entre más alto sea el porcentaje de aclaramiento, menor es el riesgo de morir ${ }^{8}$. Los autores describen valores de sensibilidad con rangos de 0.67 a 0.92 y especificidad de 0.59 a 0.75 . Ese mismo año se publica el primer trabajo realizado en pacientes pediátricos, en el que se compara el aclaramiento de lactato con una prueba pronóstica de mortalidad, pediatric risk of mortality (PRISM) por sus siglas en inglés, demostrando que el aclaramiento de lactato $<30 \%$ a las 6 horas es útil para predecir la mortalidad en pacientes pediátricos críticamente enfermos, y es inversamente proporcional a la puntuación de PRISM ${ }^{9}$. En 2016 se publica un segundo estudio pediátrico que también compara el aclaramiento de lactato con punto de corte $<16.435 \%$ a tas 6 h con otra escala pronóstica de mortalidad pediátrica, el PIM 2, y valida el aclaramiento de lactato como $\$$ rueba pronóstica útil en pacientes pediátricos críticos de cualquier patología ${ }^{10}$.

Sin embargo, ninguno de los estudios pediátricọos previamente descritos realiza comparación de làs áreas bajo la curva $(A B C)$ del aclaramiento del lactato y la prueba pronóstica de mortalidad empleada (PB̈ISM O PIM 2). Por ello, el objetivo de nuestro estudio cônsistió en determinar los valores como prueba pronóstíca del aclaramiento de lactato en pacientes pediátriços en estado crítico por cualquier etiología, utilizando êspecíficamente el punto de corte $<10 \%$ a las $6 \mathrm{~h}$, ga que este es el punto de corte referido en el estudio pionero y el más utilizado hasta el momento tanto en como en niños ${ }^{3,8,11-14}$, y compararlo con la prueba pronóstica de mortalidad pediátrica PIM 2 mediănte la prueba de Hanley \& McNeil-DeLong ${ }^{15,16}$.

\section{MÉTODOS}

\section{Diseño y población de estudio}

Se realizó un estudio prospectivo, longitudinal, o.్ర్ servacional, multicéntrico y analítico en las Unidades de Cuidados Intensivos Pediátricos (UCIP) del HospitaE్ Regional de Alta Especialidad de Ciudad Victoria y Hospital Infantil de Tamaulipas, durante el periodo compécendido del 1 de febrero de 2015 al 31 de enero de 2016. Debido a que el objetivo de este estudio fue evatiar las características de la nueva prueba (sensibilidad especificidad) y compararlas con una prueba pronóstica de mortalidad (PIM 2), se utilizó la siguiente fórmula para el cálculo de la muestra ${ }^{17}$ : 
$N=\left\{Z_{1-\alpha / 2} \sqrt{ }\left[\pi_{1}\left(1-\pi_{1}\right)\right]+Z_{1-\beta} \sqrt{ }\left[\pi_{2}\left(1-\pi_{2}\right)\right]\right\}^{2} / \delta^{2}$

$\pi_{1}$ sensibilidad PIM de $0.85^{18}$.

$\pi_{2:}$ sensibilidad esperada aclaramiento de lactato de 0.94 .

Valor alfa $(\alpha)$ : 0.05

$Z_{1-\alpha / 2}$ para el valor $\alpha: 1.96$

Valor beta $(\beta): 0.20$.

$Z_{1-\beta}$ para el valor $\beta: 0.84162$.

$\delta=\pi_{2}-\pi_{1}=0.09$.

$\mathrm{N}=105$ pacientes.

Se incluyeron pacientes de edades comprendidas entre 1 mes y 17 años con 11 meses, de cualquier diagnóstico o etiología por la que hubieran ingresado a la UCIP, que contaran con gasometría arterial a las 0 y 6 h y PIM 2 al momento del ingreso. Se excluyeron aquellos pacientes que fallecieron en las primeras $6 \mathrm{~h}$ de su ingreso, que no contaban con gasometría ni PIM 2, así como aquellos que iniciaron alguna terapia dialítica en las primeras $6 \mathrm{~h}$ de estancia. El presente estudio fue aprobado por los comités de investigación y ética en investigación de ambos hospitales, con los siguientes registros: HRAEV-IC-001-16 y HIT-INV-2015-02.

\section{Procedimientos}

Al ingreso de los pacientes se solicitó la autorización de los padres del paciente mediante la firma del consentimiento informado. Posteriormente, se obtuvo el PIM 2 de acuerdo a la fórmula descrita por Slater, et al. ${ }^{19} \mathrm{y}$ al mismo tiempo se tomó una gasometría arterial de la región de la muñeca de alguna de las dos manos, previa realización de la prueba de Allen. La muestra fue procesada en el Gasómetro ABL 700 (Radiometer Medical $^{\odot}$, Brønshøj, Dinamarca) para obtener los valores gasométricos y de lactato inicial (lactato 0). Pasadas las $6 \mathrm{~h}$ posteriores al ingreso del paciente, se tomó una nueva muestra de lactato arterial, siguiendo los mismo pasos mencionados previamente; con ambos resultados (0 y 6 h) se calculó el porcentaje de aclaramiento de lactato con la siguiente fórmula: Lactato $0 \mathrm{~h}$ - Lactato $6 \mathrm{~h} /$ Lactato $0 \mathrm{~h} \times 100^{6}$.

\section{Análisis estadístico}

Los datos se capturaron en una hoja de cálculo de Ex$\mathrm{cel}^{\odot}$ y fueron posteriormente procesados en Ios paquetes estadísticos SPSS $^{\odot}$ v.21.0 y Epidat ${ }^{\odot}$ v.3.1, ambos para Windows ${ }^{\odot}$. Los resultados de las variables ¿̇ategóricas, como sexo y defunción, se presentan comø̄ medida de frecuencia (porcentajes). Los resultados de las variables cuantitativas, como edad y días de ešstancia, se presentan como medida de tendencia central (minedia).

Para establecer la sensibilidad, especificidad, eVPN y VPP del aclaramiento de lactato se utilizó comgepunto de corte el valor de $10 \%$ a las 6 h; para ello se ellaboró una tabla de contingencia $2 \times 2$, además de hhaber calculado la razón de verosimilitud tanto positivi $\overline{\bar{a}}$ como negativa. Posteriormente, se elaboraron las corkespondientes curvas de rendimiento diagnóstico fcurvas ROC) de aclaramiento de lactato y de PIM 2 en'SPSS ${ }^{\odot}$ v.21 para Windows y se compararon mediante ba de Hanley \& McNeil-DeLong usando un vālor de $\mathrm{p} \leq 0.05$ para establecer la significancia estadística en el paquete estadístico Epidat ${ }^{\odot}$ v.3.1.

\section{RESULTADOS}

Un total de 105 pacientes pediátricos críticamentê enfermos de etiología diversa fueron estudiados en el pjeriodo estipulado, de los cuales 43 (41\%) fueron del sexo femenino y 62 (59\%) del masculino. La distribución depacientes de acuerdo con el hospital donde fueron tratados fue la siguiente: HIT con $80(76.2 \%)$ y HRAE con $25 .(23.8 \%)$ pacientes. La media de edad fue de 65.2 \pm 67.1 . La media de los días de estancia fue de $6.5 \pm 6$. 2 Hubo un total de $12(11.4 \%)$ defunciones y 93 (88.5\%) Sobrevivientes. En cuanto a los valores de aclaramiento de lactato, se obtuvo que 41 pacientes aclararon meînos del $10 \%$ y 64 pacientes aclararon más del 10\%.

Con los anteriores datos se construyó la tabla deçcontingencia de 2 × 2 y que arrojó los siguientes resátados: sensibilidad 83.3\% (IC 95\%: 58.08-100.00), espeçficidad $66.6 \%$ (IC 95\%: 56.55-76.79), índice de validez̃ $68.5 \%$ (IC 95\%: 59.22-77.93), VPP 24.39\% (IC 95\%: 10.03=38.75) y VPN 96.8\% (IC 95\%: 91.83-100.00) (Tablas 1 y

Los resultados de la curva ROC para PIM 2 fuerơn: $A B C$ de 0.88 ( $p=0.000$ ) (IC 95\%: 0.767-0.999). Los resultados de la curva ROC para el aclaramiento de lactato 
Tabla 1. Tabla de contingencia $2 \times 2$

\begin{tabular}{lccc}
\hline & \multicolumn{2}{c}{ Desenlace } & Total \\
\cline { 2 - 3 } & Muerto & Vivo & \\
\hline CL6 $<10 \%$ & 10 & 31 & 41 \\
CL6 $>10 \%$ & 2 & 62 & 64 \\
Total & 12 & 93 & 105 \\
\hline
\end{tabular}

CL6: aclaramiento de lactato a las $6 \mathrm{~h}$.

con punto de corte del 10\% fueron: $A B C$ de 0.75 ( $p=0.005)$ (IC 95\%: 0.612-0.888). Al realizar la prueba de Hanley \& McNeil-DeLong, útil para comparar áreas bajo la curva de dos o más pruebas, se obtuvo un valor de $p=0.169$ (Fig. 1).

\section{DISCUSIÓN}

El aclaramiento de lactato ha demostrado ser una adecuada prueba pronóstica de mortalidad en múltiples estudios realizados en población adulta. Recientemente se han publicado algunos estudios pediátricos tanto en pacientes con alguna patología específica, como sepsis o tetralogía de Fallot ${ }^{13,14,20}$, como en pacientes portadores de cualquier enfermedad crítica $^{9,10}$, siendo este estudio el primero en realizarse en el país en pacientes pediátricos críticos de etiología diversa ${ }^{21}$. De manera inicial, observamos en nuestros resultados que son los niños los que con mayor frecuencia enferman en comparación con las niñas, lo que coincide con lo reportado por otros autores $^{9,10}$. En cuanto a la mortalidad, nos satisface apreciar que esta fue del $11.4 \%$, la cual se encuentra dentro de los porcentajes establecidos para pacientes pediátricos críticos en Latinoamérica, que es del $13.29 \pm 7.16 \% 22$.

Algo que contrasta con otras publicaciones es la edad de nuestra muestra de estudio, debido a que en algunos estudios se limitó a edades comprendidas entre 1 mes y 13 años $^{9,10}$, y nosotros ampliamos el rango de edad de los pacientes de 1 mes a 17 años con 11 meses. Esto ofrece cierta ventaja, ya que las patologías son heterogéneas y se abarca toda la edad pediátrica estipulada en México. No se hizo análisis en cuanto a la patología que portaban a su ingreso a la UCIP, ya que no era parte del objetivo del estudio.

En cuanto a los valores de sensibilidad y especificidad referidos por los estudios pediátricos que nos preceden, tenemos los siguientes: Choudhary, et al. ${ }^{14}$ para el punto
Tabla 2. Resultados del rendimiento pronóstico del acłaramiento de lactato

\begin{tabular}{lcc}
\hline Sensibilidad & $\%$ & IC $\mathbf{9 5} \mathbf{7} \%)$ \\
& $\mathbf{8 3 . 3 3}$ & $\mathbf{5 8 . 0 8 - 1 0 0 . 0 0}$ \\
\hline Especificidad & 66.67 & $56.55-76.79$ \\
Índice de validez 0 exactitud & 68.57 & $59.22-87.93$ \\
VPP & 24.39 & $10.03-38.75$ \\
VPN & 96.88 & $91.83-100.00$ \\
RVP & 2.50 & $1.70-3.67$ \\
RVN & 0.25 & $0.07-0.89$ \\
\hline
\end{tabular}

IC 95\%: intervalo de confianza al 95\%; VPP: valor predictivo positivo; VPN. valor predictivo negativo; RVP: razón de verosimilitud positiva; RVN: razón de verosimilitud negativa.

de corte de $<10 \%$ reportan sensibilidad del $\frac{\subset}{78} .7 \%$ y especificidad del 72.2\%; Munde, et al. ${ }^{9}$ para el pünto de corte de $<30 \%$ obtuvieron sensibilidad del $75 \%$ y especificidad del 97\%; Kumar, et al. ${ }^{10}$ para un punto de corte de $<16.435 \%$ describen sensibilidad del $82.6 \%$ \& especificidad del $75.2 \%$; nuestros valores fueron senșibilidad del $83.3 \%$ y especificidad del $66.6 \%$. Como se aprecia, los valores son heterogéneos y todos se ubicañontentro de los rangos descritos por Zhang, et al. ${ }^{8}$, los cuatés son: sensibilidad del 67 al 92\% y especificidad del 59 al $75 \%$.

Anteriormente se demostró el lactato sérico indidividual al ingreso a UCIP como un factor predictor de Bortalidad independiente del PIM $2^{23}$. Por ello, a diferencia de Kumar, et al. ${ }^{10}$, que mencionan un $\mathrm{ABC}$ de 0.82 yara el aclaramiento de lactato y $\mathrm{ABC}$ de 0.90 para $\mathrm{PIM}^{-2}$, pero no realizaron alguna prueba estadística que permitiera establecer una diferencia entre ambas. En nuestro estudio se realizó la comparación de las $\mathrm{ABC}$ tânto del aclaramiento de lactato $<10 \%$ a las 6 h como êde PIM 2 mediante la prueba de Hanley \& McNeil-DeEong, y evidenciamos que no existe diferencia estadística entre ambas pruebas (Fig. 1).

Consideramos con estos resultados que el aclaramiento de lactato, con un punto de corte de $<10 \%$ a. a $6 \mathrm{~h}$, es una prueba que posee aceptables valores dênsensibilidad, especificidad y área bajo la curva que lẩh hacen útil para pronosticar mortalidad en pacientes peđjátricos mexicanos en estado crítico por cualquier etiología.

Recomendamos que se utilice el aclaramiento gle lactato como valor evolutivo junto con los marcadöres de uso habitual, los hallazgos clínicos y escalas pronósticas de mortalidad, ya que permite anticiparnos-a un resultado catastrófico e instalar de manera temprana 


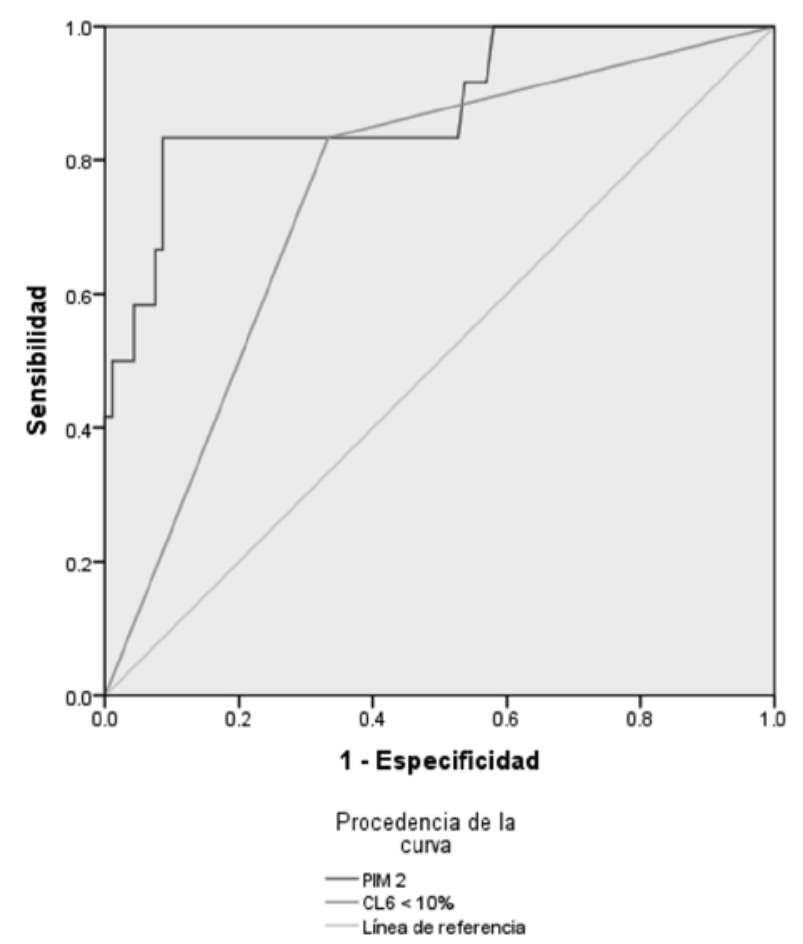

Figura 1. Prueba de Hanley \& McNeil-De Long para comparar ambas áreas bajo la curva $(p=0.169)$. CL6: aclaramiento de lactato a las 6 h; PIM 2: índice pediátrico de mortalidad.

estrategias para lograr revertirlo. Si con una terapia intensiva conseguimos el descenso de los valores de lactato, quiere decir que debemos continuar con las medidas establecidas e intensificarlas. $\mathrm{Si}$, por el contrario, los valores continúan incrementándose, es necesario revisar el tratamiento y valorar si el problema inicial está solucionado o si la gravedad del enfermo es tan importante que la situación es irreversible ${ }^{3,6}$.

\section{FINANCIAMIENTO}

Estudio realizado, aprobado y financiado por el Hospital Regional de Alta Especialidad de Ciudad Victoria y el Hospital Infantil de Tamaulipas.

\section{BIBLIOGRAFÍA}

1. Bermúdez-Rengifo WA, Fonseca-Ruiz NJ. Utilidad del lactato enfel paciente críticamente enfermo. Acta Colomb Cuid Intensivo. 2016;16(2):80-9.

2. Bai Z, Zhu X, Li M, Hua J, Li Y, Pan J, et al. Effectiveness of predicting in-hospital mortality in critically ill children by assessing blood lactate levels at admission. BMC Pediatr. 2014;14:83.

3. Nguyen BH, Rivers PE, Knoblich BP, Jacobsen G, Muzzin A, Ressler JA, et al. Early lactate clearance is associated with improved outcome-in severe sepsis and septic shock. Crit Care Med. 2004;32(8):1637-42.

4. Cardinal-Fernández PA, Olano E, Acosta $\mathrm{C}$, Bertullo $\mathrm{H}$, Albornoz-H, Bagnulo $\mathrm{H}$. Valor pronóstico del aclaramiento de lactato en las primeras $6 \mathrm{~h}$ de evolución en medicina intensiva. Med Intensiva. 2009:33(4):166-70.

5. Jansen TC, van Bommel J, Schoonderbeek FJ, Sleeswijk Visser SJ, van der Klooster JM, Lima AP, et al.; LACTATE study group. Early lactate-guided therapy in intensive care unit patients. Am J Respir Crit Care Med. 2010; 182(6):752-61.

6. Pasha A, Rao N, Prabodh S, Sripad DV, Chowdary NV. Relationship between serum lactate levels and fatal outcome in critically ill patients: A prospective study in intensive care unit. Int J Sci Stud. 2015;2(10):61-3.

7. Porras-García W, Ige-Afuso M, Ormea-Villavicencio A. Depuración de lactato como indicador pronóstico de mortalidad en pacientes eon sepsis severa y choque séptico. Rev Soc Peru Med Interna. 2007;20(4):132-8.

8. Zhang Z, XuX. Lactate clearance is a useful biomarker for the prediction of all-cause mortality in critically ill patients: a systematic review $\overline{\text { and }}$ metaanalysis. Crit Care Med. 2014;42(9):2118-25.

9. Munde A, Kumar N, Beri RS, Puliyel JM. Lactate clearance as a-marker of mortality in pediatric intensive care unit. Indian Pediatr. 2014;51-67):565-7.

10. Kumar R, Kumar N. Validation of lactate clearance at $6 \mathrm{~h}$ fo Pmortality prediction in critically ill children. Ind J Crit Care Med. 2016;20(40):570-4.

11. Saldaña-Vázquez R, Hernández-Portales J, Ramírez-Rosales A. Depuración de lactato como marcador pronóstico en pacientes con sepsis severa y choque séptico en la UCI. Rev Asoc Mex Med Crit y Ter Int. 2012;26(4):194-200.

12. Kittivoravitkul $P$, Wattanathum $A$, Wongsa $A$. Lactate and lactate clearance were associated with higher mortality in patients with septic.shock. Eur Resp J. 2011;38:1993.

13. Ladha S, Kapoor PM, Singh SP, et al. The role of blood lactate clearance as a predictor of mortality in children undergoing surgery for tetralogy of Fallot. Ann Card Anaesth. 2016;19:217-2.

14. Choudhary R, Sitaraman S, Choudhary A. Lactate clearance as the predictor of outcome in pediatric septic shock. J Emerg Trauma Shock. 2017; $10: 55-9$

15. Hanley JA, McNeil BJ. A method of comparing the areas under receiver operating characteristic curves derived from the same cases. Radiology. 1983 148:839-43.

16. DeLong ER, DeLong DM, Clarke-Pearson DL. Comparing the areas under two or more correlated receiver operating characteristic curves:-a nonparametric approach. Biometrics. 1988;44:837-45.

17. Sánchez- Pedraza R, Echeverry-Raad J. Aspectos sobre diseñoy tamaño de muestra en estudios de pruebas diagnósticas. Rev Fac Medicina (UNAM, México). 2001;49(3):175-80.

18. Prieto-Espuñes S, López-Herce Cid J, Rey-Galán C, Medina-Vithanueva A, Concha-Torre A, Martínez-Camblor P. Índices pronósticos de mortalialidad en cuidados intensivos pediátricos. An Pediatr (Barc) 2007;66(4):345-50.

19. Slater A, Shann F, Pearson G. PIM 2: a revised version of the 'Paediatric Index of Mortality. Intensive Care Med. 2003;29:278-85.

20. Kim YA, Ha EJ, Jhang WK, Park SJ. Early blood lactate area as æprognostic marker in pediatric septic shock. Intensive Care Med. 2013;39:1818-23.

21. Lara-Hernández CA, Fuentes-Mallozzi DA. Resúmenes de tesis de Jos cursos de especialización médica: Validez del aclaramiento de lactato enpacientes pediátricos graves. Evid Med Invest Salud. 2016;9(Supl 1):19.

22. Campos-Mino A, Sasbón JS, von Dessauer B. Los cuidados èntensivos pediátricos en Latinoamérica. Med Intensiva. 2012;36(1):3-10." ¿

23. Morris KP, McShane P, Stickley J, Parslow RC. The relationship between blood lactate concentration, the Paediatric Index of Mortality 2 ( $\mathrm{EM} 2$ ) and mortality in paediatric intensive care. Intensive Care Med. 2012;38:2042-6. 